\title{
La cultura tradicional leonesa en la obra de Julio Caro Baroja
}

En una tarde de mayo, hace ahora quince años, escuché a don Julio una conferencia magistral que tituló "En torno a la Etnología leonesa" ": una síntesis clara de lo que pensaba y, en ocasiones, había escrito sobre la cultura tradicional de las comarcas leonesas. Nos descubrió y recordó con cariño a algunos de sus informantes leoneses, y en especial a aquella tertulia madrileña del viejo Café Varela donde se reunían comerciantes de la Maragatería, pescaderos y carniceros, que le contaron cosas de interés, efundamentalmente - decía él- para comprender algunos aspectos de ese mundo de las fiestas rituales de Españaw. Don Julio era amigo de las tertulias, como lo fue su tío Pío y otros hombres del 98, gustaba de conversar con los amigos sobre los más variados temas, costumbre que mantuvo casi hasta el final de su vida.

Otra persona que - según él- amplió su visión de la etnografía leonesa fue el escritor y musicólogo, Manuel Fernández Núñez (La Bañeza 1883-Madrid 1952), buen conocedor de la música popular, y al que recordó con gran afecto ${ }^{2}$.

No llegó a conocer personalmente a José Aragón Escacena ${ }^{3}$, pero esa tarde valoró mucho su novela costumbrista Entre brumas (Astorga, 1921) que encontró en la biblioteca de su tío Pío Baroja, y que le supuso un precioso hallazgo, ya que en ella se describen costumbres y tradiciones de la Cabrera Baja, comarca visitada también durante el invierno de los años 1921-1922 por el profesor Fritz Krüger con motivo de la investigación de campo que estaba realizando sobre la cultura popular de Sanabria y sus zonas limítrofes.

1 El 4 de mayo de 1981 en la Casa de León, en Madrid. Conferencia publicada en la Revista de la Casa de León, Verano (1981), pp. 3-9.

2 Entre las obras de FERNÁNDEZ NÚÑEZ, Cantos populares leoneses (1909), Folklore bañezano (1914), Folklore leonés (1935). Cf. Diccionario bistórico de la Antropología española (Madrid: C.S.I.C., 1994), pp. 304-305.

3 Según datos que me facilitó don Augusto Quintana, nació en Astorga h. 1895 y murió allí en 1945. Es autor también de alguna obra de teatro y libro de cuentos. Su novela Entre brumas obtuvo el Primer Premio en el concurso del IX Centenario de los Fueros de León, año 1920. 
¿Qué es León para don Julio?

León es en esencia un contorno medieval, un producto de la Reconquista, León no hace referencia a un grupo étnico, como, por ejemplo, Asturias (tierra de astures) o Cantabria (de cántabros), sino a una capitalidad, a una ciudad, y sobre esto "se hace como el meollo, el cogollo de lo que es lo leonés".

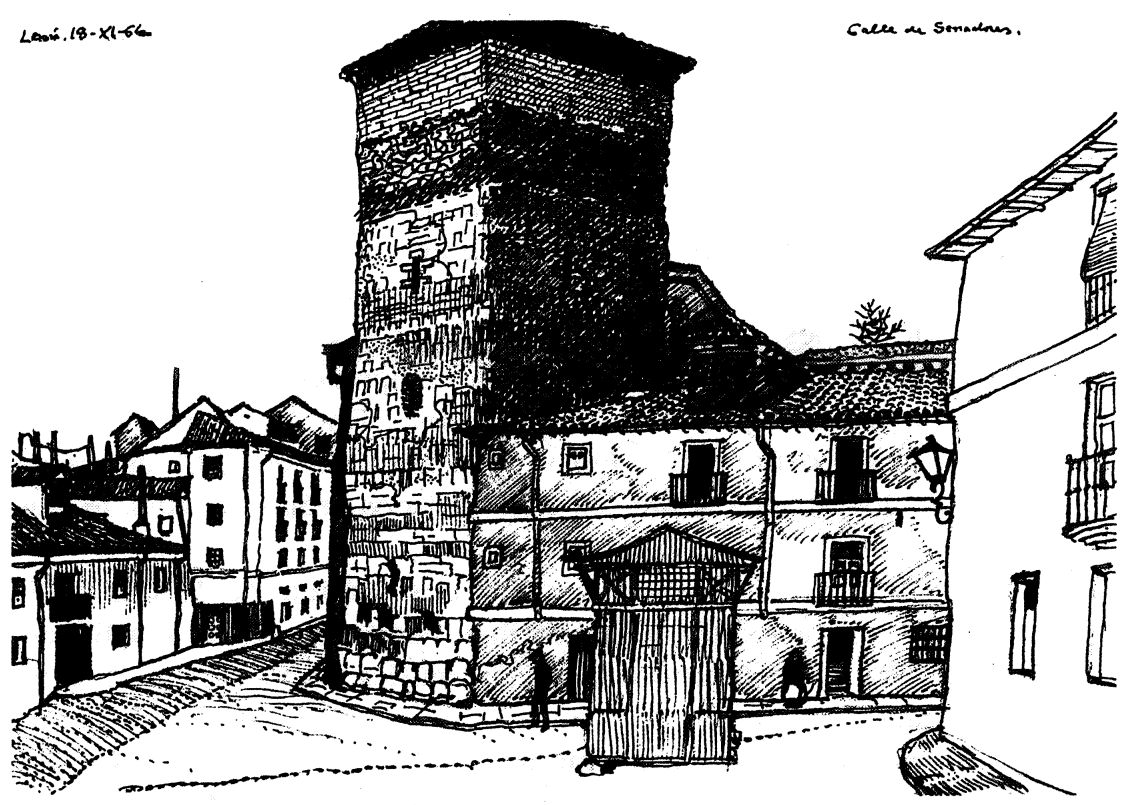

FIG. 1.-León. Calle de Serradores. Dibujo de Julio Caro Baroja, tomado del natural el 18-XI-1964.

León es un conjunto de comarcas, que podrían agruparse fundamentalmente en tres: montaña, vega y páramo (o lo que José Luis Alonso Ponga denominaría más tarde la tierra llana):

No podemos hablar de que León sea una comarca natural en el sentido que los geógrafos físicos hablan de comarca natural; es más que una comarca natural, es un conjunto de comarcas, y es una unidad de tipo más bien político en un momento, y esta condición de unidad política, con su capitalidad, le va dando estructuras nuevas y le va dando una especie de relaciones o de organizaciones nuevas ${ }^{4}$.

Léon es para don Julio una de las tierras de España más ricas en variedades culturales; aunque tenga esta unidad política vieja y su folklo-

4 Conferencia citada en la nota núm. 1, pp. 5-6. 
re responda a un conjunto de lo que es el folklore euro-occidental, le da a todo un sello particular.

La cultura material de las comarcas leonesas es - según él- de una riqueza, de una variedad y de un valor informativo grandes para todo lo que es la historia de la tecnología. Merecieron su atención los tipos de $\operatorname{arados}^{5}$ y de carros, así como esa especie de narria, el forcao, de la comarca de Gordón ${ }^{6}$, y a ellos dedica no solo un comentario sino que también hizo un dibujo, por ejemplo, del modelo de rueda que aparece en el retablo de la catedral leonesa, atribuido a Nicolás Francés, siglo XV.

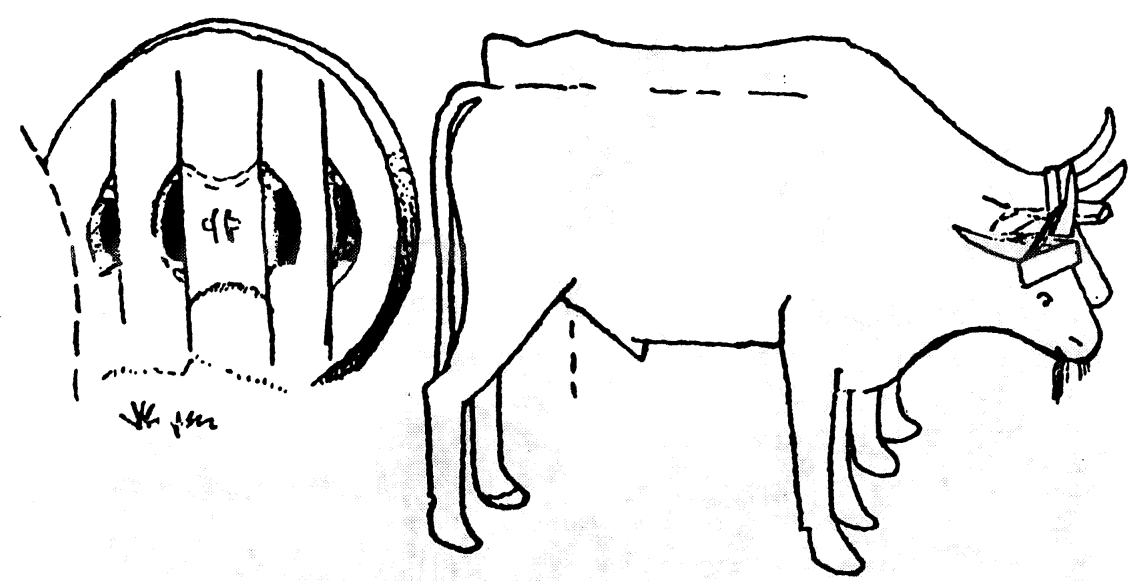

FIG. 2.-Rueda maciza de las carretas leonesas, representada en una tabla del altar mayor de la catedral de León, pintado por Nicolás Francés.

Recuerdo su cara de sorpresa cuando vio en unas acuarelas del año 1926 este mismo tipo de rueda, que pintaron en la Maragatería los alumnos de la Escuela Madrileña de Cerámica durante su estancia veraniega en el pueblo de Val de San Lorenzo. Todavía sigue usándose en la Cabrera $^{7}$, y el viajero alemán Hans Gadow la fotografió a finales del siglo XIX en Riaño ${ }^{8}$.

5 Y su distribución por la geografía leonesa, en Tecnología popular española (Madrid: Ed. Nacional, 1983), pp. 543 y ss.

6 Cita el trabajo de J. DANTín CERECEDA, publicado en AMPE, I (1935), pp. 139-148.

7 Para más detalles, cf. el estudio de José Luis ALONSO PONGA, Los carros en la agricultura de Castilla y León (Valladolid: Junta de Castilla y León, 1994), con abundantes ilustraciones.

8 Cf. C. CASAdo y A. CARREIRA, Viajeros por León. Siglos XII-XIX (León: Santiago García editor; 1985), p. 309. 
Hay una idea de don Julio, escrita en 1946, que me gustaría destacar. Pensaba él que "difícilmente se podrá encontrar en Europa una región en la que los elementos de la cultura moderna se hallen tan en armonía con

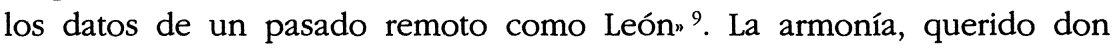
Julio, se nos va resquebrajando por no saber valorar todo el rico patrimonio heredado. Es triste ver cómo desaparecen y cómo se sustituyen esas muestras de nuestra arquitectura, por poner un ejemplo.

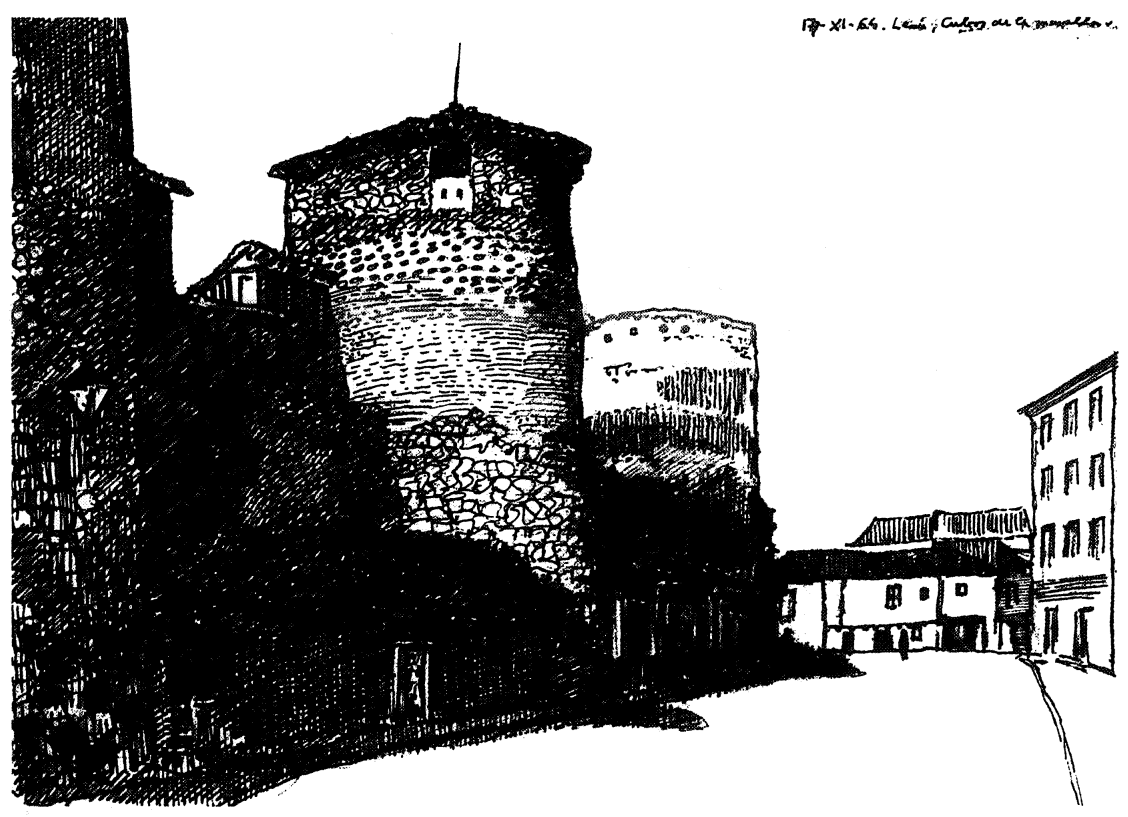

Fig. 3.-Muralla de León. Vista de los cubos de la muralla, dibujado el 17-XI-1964 durante una estancia de trabajo en la ciudad.

A las singulares tipologías de la arquitectura popular leonesa consagró breves páginas en su obra Los Pueblos de España ${ }^{10}$. Se detiene en analizar las arcaicas construcciones de la zona de Riaño (en la montaña oriental), la casa semicircular de las comarcas de Babia y Laciana (montaña occidental) y las construcciones con tapial y adobe (del páramo leonés) ${ }^{11}$.

\footnotetext{
9 Los Pueblos de España (Madrid: Ediciones Istmo, 1976), I, p. 67 (la primera edición es de 1946).

10 Op. cit. en la nota anterior, pp. 62-64.

11 Hace referencia a las obras de Cárdenas (1944), Modesto Medina Bravo (1927) y Gustavo Fernández Balbuena (1922).
} 
Recientemente han aparecido estudios más amplios sobre la arquitectura popular leonesa, como el del arquitecto José Luis García Grinda o del etnógrafo José Luis Alonso Ponga ${ }^{12}$.

Es quizás en sus trabajos sobre el ciclo festivo anual donde podemos encontrar más abundantes referencias a las comarcas leonesas. Las fuentes impresas utilizadas en relación con las fiestas tradicionales de la provincia de León fueron, principalmente, las obras de Santiago Alonso Garrote ${ }^{13}$ y de Matías Rodríguez ${ }^{14}$, para las tierras de Astorga, y la novela costumbrista de José Aragón Escacena para la Cabrera. El P. César Morán le proporcionó interesantes descripciones de las fiestas en Omaña, y Dolores Álvarez Prida algunos datos importantes sobre danzas de paloteo en el Bierzo.

Los libros que publicó sobre fiestas, elaborados a partir de materiales recogidos para su tesis doctoral hace más de cincuenta años, contienen una documentación excepcional y en su conjunto son algo difícil de superar. Cabe, sin embargo, complementarlos con observaciones y estudios puntuales a los que don Julio no tuvo acceso o que aparecieron con posterioridad.

En 1949, doña Dolores Álvarez Prida, de familia ponferradina, le envió un detallado informe acerca de los danzantes que salían en Ponferrada por la fiesta de Nuestra Señora de la Encina, patrona de esta ciudad. Ese día, 8 de septiembre, iban en la procesión los danzantes, con el Calaverote o gracioso. En el momento de salir la Virgen de la iglesia, los danzantes formaban una pirámide compuesta de cuatro en base, tres sobre los hombros de éstos y uno encima, que recitaba una alabanza a Nuestra Señora de la Encina ${ }^{15}$. En la procesión, iban bailando a los sones del tamboritero y de una enorme castañuela que tocaba el Calaverote. Finalizada la procesión, danzaban nuevamente en torno a un palo vertical, de cuyo extre-

12 J. L. GARCía GRINDA, Arquitectura Popular Leonesa (León: Diputación Provincial, 1991), 2 vols., y J. L. ALONSO PONGA, La arquitectura popular leonesa (León: Caja León, 1990), y La arquitectura del barro (Valladolid: Junta de Castilla y León, 1986) (3.a ed., 1994).

13 El dialecto vulgar leonés bablado en Maragatería y tierra de Astorga (Madrid: C.S.I.C., 1947, 2. ${ }^{\mathrm{a}}$ ed.).

14 Historia de la muy noble, leal y benemérita ciudad de Astorga (Astorga: Porfirio López, 1909).

15. Curiosamente, en 1976, con motivo del VIII Centenario de la fundación del Monasterio Cisterciense de Carrizo en el pueblo se volvió a revivir la antigua danza de la fiesta patronal, Nuestra Señora del Villar (martes de Pentecostés) y presencié cómo formaban también los ocho danzantes de Carrizo una torre humana, que me sorprendió un poco porque no lo había visto hacer en otros pueblos leoneses. 
mo superior pendían unas cintas que iban entretejiendo. La indumentaria de los danzantes y otros datos quedaron reflejados por don Julio en su libro $E l$ estío festivo ${ }^{16}$. Es un texto muy interesante, puesto que se ha perdido ya esta danza en Ponferrada, a la que también aludía brevemente un escritor del pasado siglo ${ }^{17}$.

Ya en 1934, recoge Verardo García Rey, en su Vocabulario, la figura del Caravelote [sic] o gracioso que formaba parte de la danza en Molinaseca ${ }^{18}$. Hoy tan solo queda el recuerdo. No así en el valle berciano de Fornela donde perviven los danzantes en las fiestas de Nuestra Señora de Trascastro, el 15 de agosto.

Un precioso texto, poco conocido, sobre danzas bercianas es el del poeta Antonio Fernández y Morales que nos describe la fiesta de San Roque en Paradela del Río (pueblo cercano a Corullón), donde junto a los ocho danzantes figura también el Caravelote y, otro personaje, la Dama ${ }^{19}$. Tanto aquí como en Ponferrada y Molinaseca se acompañaba la danza con música de flauta y tamboril.

Estas danzas, que se enmarcan dentro de un rito o ceremonia, han estado muy extendidas por tierras leonesas. Son coreografías ejecutadas por hombres y tenían lugar, principalmente, en la festividad del Corpus Christi. Y son famosas todavía las danzas del Corpus en Laguna de Negrillos y las de San Esteban de Nogales, en la festividad de San Jorge, el 23 de abril. En el pueblo de Corporales (Cabrera Alta) tenemos la "Danza del rey Nabucodonosor", en la festividad del Corpus, que incluía antes una representación teatral con ese tema bíblico ${ }^{20}$. También por la Maragatería, el Bajo Órbigo, y otros lugares leoneses, se recuerdan este tipo de danzas y las familias conservan material gráfico de ellas, e incluso algunos músicos locales tienen en su repertorio las melodías, como el tamboritero de Rabanal del Camino, el dulzainero de Laguna de Negrillos o el gaitero de Corporales de Cabrera, entre otros ${ }^{21}$.

16 El estío festivo (fiestas populares del verano) (Madrid: Taurus, 1984), pp. 161-162.

17 Citado también por don Julio, Acacio CÁCERES PRAT, El Vierzo, su descripción e bistoria, tradiciones y leyendas (Madrid, 1883), pp. 129-130.

18 En la danza de Molinaseca intervenían ocho danzantes, el Caravelote o gracioso, un joven vestido de mujer y el tamboritero. Como partes de la danza: juego de palos (o paloteado), banderas y cintas. Cf. Verardo GARCía REY, Vocabulario del Bierzo (Madrid, 1934), s. v. Caravelote.

19 Antonio FERNÁNDEZ Y MORALES, Ensayos poéticos en dialecto berciano (León: Imprenta Miñón, 1861), pp. 243-255.

20 Cuyo texto publiqué en la revista Tierras de León (1993), pp. 138-148.

21 Cf. C. CASAdo Lobato, Folklore, en León (Madrid: Ed. Mediterráneo, 1989, pp. $172-176$. 
De las fiestas en los meses de mayo y junio destaca don Julio el mayo y la noche de San Juan. Para el mayo recoge documentación en los libros de Santiago Alonso Garrote ${ }^{22}$ y de Matías Rodríguez ${ }^{23}$, que ofrecen datos sobre las tierras de Astorga. Un amigo suyo del pueblo de Canales (cercano a La Magdalena) le contó cómo se celebraba la fiesta de la colocación del mayo hacia 1914. Comenta también un texto de Elías López Morán ${ }^{24}$ con referencia al árbol-mayo que colocaban en los pueblos por las fiestas de San Juan y San Pedro, haciendo hincapié en el gallo que colgaban (en lugar del muñeco) y en las peticiones y rondas de los mozos.

La colocación del mayo estuvo muy generalizada en tierras leonesas y todavía se mantiene. En el pueblo alfarero de Jiménez de Jamuz los mayos son muñecos que representan personajes del pueblo (o que tuvieron alguna relación con él) y cada barrio sigue organizando su mayo.

En la montaña oriental y en el Alto Esla se acostumbra a colocar "el mayo del misacantano", y es un hecho que presenció en el pueblo de Riaño, ya a fines del siglo xIx, el viajero alemán Hans Gadow y que comenta así en su diario: "Hay la excelente costumbre de que un joven misacantano visite su pueblo natal y celebre una misa junto al mayo erigido por sus antiguos compañeros, a fin de dejar memoria del honor conferido a su pueblo" 25 .

En algunos lugares de las montañas de Villafranca del Bierzo, cercanos a Galicia, el mayo era un muchacho que salía cubierto con ramas de escoba; él y sus compañeros iban cantando y pidiendo por las casas. El carácter de fiesta de la vegetación se hace aquí más evidente.

Sobre la noche de San Juan en las comarcas leonesas se centra principalmente en unas páginas de José Aragón Escacena, comentando que en la Cabrera Baja llevaban esa noche a bañar los ganados al río. Recoge don Julio -como es habitual en él- muchos y valiosos datos de toda España sobre la creencia del poder curativo del agua y del rocío en esa noche ${ }^{26}$.

A la fiesta del arado que se celebraba el primero de año en la Alta Maragatería, le dedica una atención especial. La primera descripción de

\footnotetext{
22 Op. cit. en nota 13.

23 Op. cit. en nota 14 .

24 Derecho consuetudinario leonés (1. ${ }^{a}$ edic. 1902).

25 Cf. Viajeros por León, op. cit. en nota 8, p. 34.

26 Cita también don Julio un curioso texto de Madoz sobre las Cuevas de San Genadio (en el Bierzo) a las que por San Juan se iba a recoger polvo de ellas que suponen específico contra las calenturas intermitentes...”, Cf. Diccionario Geográfico, s. $v$. Cuevas de San Genadio.
} 
esta fiesta, tal como se hacía por los años veinte, la escuchó en esa tertulia de maragatos del viejo Café Varela; luego buscó información en la obra de Santiago Alonso Garrote ${ }^{27}$ y, más tarde, le completarán esos datos dos personas que habían participado activamente en esta fiesta singular, Jesús Argüello y su hermano, de Rabanal del Camino, quienes le hablaron de cómo se celebraba la fiesta del arado en los pueblos de la Alta Maragatería, cercanos al Teleno: la noche de Año Viejo salían a rondar los zamarracos (solían ser seis) vestidos con pieles de ovejas y acompañados de la Dama. Todos llevaban cubierto el rostro; la dama, careta de rostro femenil y los zamarracos de viejos barbudos. Uno de éstos personajes llevaba un palo a modo de tenedor o tridente para enganchar los trozos de tocino, embutidos, etc. que los vecinos les iban regalando. Pero es el día primero de año cuando tienen lugar los festejos más importantes. Por la mañana, a la salida de misa y en el pórtico de la iglesia, los zamarracos, por turno, van recordando los acontecimientos del pueblo durante el año. Al final, la Dama y uno de los zamarracos interpretan un diálogo en verso a modo de comedia. Por la tarde, se realiza propiamente la fiesta del arado: "generalmente sobre la nieve, los zamarracos tiran de un arado que guía la dama, y siembran o hacen que siembran". Después bailan todos los del pueblo, que quieran hacerlo, al son del tamboril. Analiza minuciosamente todos los detalles y comenta aquellos aspectos más significativos de esta fiesta, que parece ser se hacía con el fin de asegurar las cosechas y la fecundidad de las ovejas. Esta fiesta del arado tiene semejanzas en otras culturas. En casi toda Europa y también en países más lejanos —escribía él- persisten costumbres como la de Maragatería ${ }^{28}$.

Para la celebración de los Antruejos o Carnavales, su buen amigo el P. César Morán (Rosales 1882-Madrid 1952) le facilitó información sobre la zafarronada de los pueblos de Omaña e incluso algún material gráfico ${ }^{29}$. Ya el P. Morán había publicado en 1931, en la prestigiosa revista madrileña del Museo Antropológico, una descripción de esta fiesta tal como solía hacerse a finales del pasado siglo ${ }^{30}$. Los datos son de sumo interés

27 Op. cit. en nota 13.

28 Cf. El Carnaval (Madrid: Taurus, 1979), po. 286-288 (la primera edic. es de 1965). Isabel BOTAS SAN MARTín, oriunda de Santa Marina de Somoza en la Alta Maragatería, recoge esta fiesta de pastores en su libro, La Maragatería (Madrid, 1993), pp. 27-31 (cito por la 2. ${ }^{a}$ edic., la primera es de 1990).

29 En la 1. ${ }^{a}$ edic. de El Carnaval (1965) se reproduce una fotografía del zafarrón de Rosales, que le envió el P. Morán.

30 "Datos etnográficos", Sociedad de Antropología, Etnografía y Prebistoria. Actas y Memorias, X (1931).p 
y don Julio destaca la relación de la zafarronada omañesa con las mascaradas suletinas, las gallegas de Cotobade y Viana, así como con los guirrios asturianos ${ }^{31}$.

También de tierras bañezanas incluye la aportación de don Manuel Fernández Núñez sobre "los juanillos", mozos que salían por las calles durante los tres días de Carnaval disfrazados de vaquillas, con una colcha y un par de cuernos. Del tema de las "vaquillas", en 1936, le facilitó una joven, oriunda de Rebollar de los Oteros (en la zona de Valencia de Don Juan), la descripción de la fiesta que en este pueblo se hacía el día de San Blas: dos hombres, cubiertos con una manta parda y unos cuernos, imitan ser una vaca; otros, disfrazados con grandes cencerros la siguen por las calles del pueblo. Al día siguiente, que llaman de "San Blasín", hacen como que matan a "la vaca" y beben su sangre, es el vino que reparten los mozos ${ }^{32}$. En algunos pueblos de la Ribera del Orbigo siguen celebrándose los Antruejos tradicionales, como los toros y guirrios en Velilla de la Reina y Llamas de la Ribera ${ }^{33}$.

Una breve reseña sobre la Candelaria, el día 2 de febrero, le facilitó don Manuel Fernández Núñez ${ }^{34}$, donde se contemplan las ofrendas de palomas, panes y velas que se hacían en esa festividad. Por la Candelaria es costumbre la bendición de candelas y el ofrecimiento de palomas. La Cofradía de los "Usías", en la ciudad de León, parroquia de Santa Marina, mantiene año tras año la tradición ${ }^{35}$.

Otros temas de la etnografía leonesa que merecieron la atención de don Julio fueron la arriería y la transhumancia, el colectivismo agrario, las sociedades de mozos y el papel de la mujer en la vida rural.

Muy brevemente trata la arriería de los maragatos y sus costumbres ${ }^{36}$ La arriería - como es sabido- no fue exclusiva de los maragatos dentro de la provincia de León, había arrieros en la comarca de Los Argüellos (La Vecilla) y en varios pueblos de Laciana y Babia (Murias de Paredes), cercanos a Asturias; unos y otros hacían la ruta entre Asturias y Castilla.

31 Cf. El Carnaval, pp. 230-231.

32 Hace unos años se recordaba por personas mayores del pueblo este festejo. Recoge también don Julio un dato sobre disfraces de bóvidos en la Cabrera Baja, que aparece en la obra de León MARTín Granizo, La Provincia de León. Paisajes, hombres, costumbres y canciones (Madrid: Juan Ortiz editor, 1929), p. 43.

33 Sobre los "toros y guirrios" de Velilla de la Reina publiqué unas notas en mi artículo Folklore del libro León, op. cit. en nota 21, p. 166.

34 Cf. nota 2.

35 Cf. en nota 21, p. 162

36 Cf. Los pueblos de España, op. cit., nota 9, I, p. 67. 
Los maragatos transportaban, sobre todo, mercancías entre Galicia y Castilla. En el siglo xviI hay un gran crecimiento y expansión de la arriería maragata y los viajeros de esa época nos dejaron interesantes testimonios en sus relatos ${ }^{37}$.

Sobre el origen del pueblo maragato se han propuesto diversas teorías. Unos se inclinaron a ver en ellos a lejanos descendientes de beréberes o norteafricanos, así Dozy, Aragón Escacena, Alonso Garrote y, más tarde, Oliver Asín. Otros, siguiendo a Caro Baroja, les consideran un grupo social y etnográfico de origen astur con tendencias muy conservadoras, ya que el habla de esta comarca presenta los rasgos característicos del leonés occidental y sus técnicas agrícolas, aperos de labranza, etc., son semejantes a los de las vecinas comarcas; sí hay que destacar entre ellos una rígida endogamia.

La transhumancia leonesa, bien estudiada —según él- desde el punto de vista de las cañadas, lo está menos desde el etnográfico, aunque puede haber dejado huellas en el norte de Huelva ${ }^{38}$.

Para el tema de la propiedad colectiva en algunos pueblos leoneses, resume el estudio que realizó Elías López Morán ${ }^{39}$ a comienzos de siglo, y enumera algunos ejemplos significativos, como puede ser la bouza en pueblos de La Cabrera, terreno donde los vecinos del lugar aran, siembran, siegan, extraen y limpian el grano, todo en común (el comienzo de las faenas se determina en concejo de vecinos). Otras formas de explotación comunal recordadas son "los préstamos" de Gusendos de los Oteros, "la ronda" de Valdemora, "las vitas" de Sahagún, el reparto de tierras en Llánaves, etc. ${ }^{40}$

Teniendo también como principal fuente documental los estudios de Elías López Morán, se detiene en analizar la división tradicional de los habitantes de una aldea —al menos, hasta comienzos de siglo-: niños, rapaces, mozos y vecinos, con las características y cometidos de cada uno

37 Cf. José Luis GALINDO, Arrieros maragatos en el siglo XVIII (Valladolid: Universidad de Valladolid), 1956, y Laureano RUBIO PÉREZ, Arrieros maragatos. Poder, negocio, linaje y familia. Siglos XVI-XIX (León: Fundación Hullera Vasco-Leonesa, 1995). Sobre relatos de viajeros, op. cit., nota 8 .

38 «En la provincia de Huelva en el año cincuenta, cuando yo estuve, era posible encontrar cosas interesantes para el etnógrafo que no podían ser más que de origen septentrional, de origen leonés". Cf. op. cit., nota 9, I, p. 67.

39 Op. cit., nota 24 .

40 Op. cit., nota 9, p. 65 y Elías LÓPEZ MORÁN, Derecho consuetudinario leonés, cito por la edición de 1984, Colección "Breviarios de la calle del Pez", Diputación Provincial de León, pp. 35-45 (la primera edición es de 1902). 
de estos cuatro grupos. A la sociedades de mozos y sus funciones, dentro del mundo rural, les dedica un interesante comentario ${ }^{41}$.

El trabajo de la mujer en el campo, cuyo estudio había abordado ya Elías López Morán es también recogido por don Julio, así como ciertos aspectos relacionados con el matrimonio en las zonas rurales ${ }^{42}$.

Como hemos visto, varios son los temas de la etnografía leonesa que podemos encontrar en las obras de Julio Caro Baroja, cuando las escribió todavía la cultura tradicional seguía viva en muchos lugares. Sin embargo, ya en 1981 -comentaba él en la conferencia que citamos al principiosólo quedaba el recuerdo, pero este recuerdo era decisivo, porque son las gentes de cierta edad, las personas mayores, "las que nos pueden dar la visión de un mundo perdido ya para la gente joven", y hacía un llamamiento a los estudiosos de la cultura tradicional para que recogieran las reliquias del pasado, "un pasado que, muchas veces, es de una riqueza increỉblen.

\title{
CONCHA CASADO LOBATO \\ Departamento de Antropología Instituto de Filología. CSIC
}

\begin{abstract}
Se estudian pormenorizadamente los informes, fuentes bibliográficas y datos de trabajo de campo acerca de la cultura popular leonesa, incluidos en las obras de conjunto más importantes publicadas por Caro Baroja. Se insiste en los aspectos más significativos de algunos rituales, grupos específicos como los maragatos, tecnología, etc., que ayudan a la comprensión de la identidad y la variedad, características de lo leonés.
\end{abstract}

The author analyzes in detail the reports, bibliographies, and fieldwork data on Leon's folk culture contained in Julio Caro Baroja's most important general publications. It focuses on the most significant aspects of some rituals, specific groups such as the Maragato, technology, and other traits that help understand the identity and characteristic variety of Leonese culture.

41 Cf. Los pueblos de España, op. cit., nota 9, pp. 68-69 y El Carnaval, op. cit., nota 28, p. 339.

${ }_{42}$ Sobre el tema puede consultarse el trabajo de Prisciliano CORDERO DEL CASTILLO, "La familia rural leonesa. (Un sistema llamado a desaparecer)", Tierras de León, 32-33 (1978), pp. 88-103. 